There was a trend to less proximal reflux events with the alginate compared to the antacid (10.5 (8.9) vs 13.9 (8.3), $\mathrm{p}=0.070)$. No difference in the number of symptoms (5.0 (6.0) vs 4.2 (8.3), $\mathrm{p}=0.701)$ and reflux related symptoms (2.5 (4.0) vs 2.8 (5.6), $\mathrm{p}=0.988$ ) was reported.

Conclusion Standard $\mathrm{pH}$-impedance monitoring is suitable for clinical studies of reflux suppression in GORD patients. Distal reflux was similar but a trend to suppression of proximal reflux by GA compared to $\mathrm{MM}$ is present. This feasibility data indicates that trials will require 70 GORD patients to demonstrate effects (power 90\%, p<0.05) on proximal reflux suppression after meals by alginates. More prolonged studies are required to assess effects on symptom control.

Competing interests R Sweis: None declared, A Anggiansah: None declared, $T$ Wong: None declared, M Fox Grant/Research Support from: Reckitt Benckiser.

\section{PWE-059 PREVALENCE OF GASTRO-ESOPHAGEAL REFLUX DISEASE IN INDIVIDUALS WITH IRRITABLE BOWEL SYNDROME: A SYSTEMATIC REVIEW AND META-ANALYSIS}

doi:10.1136/gutjnl-2012-302514d.59

${ }^{1} \mathrm{R}$ M Lovell, ${ }^{* 1,2} \mathrm{~A}$ C Ford. 'Leeds Gastroenterology Institute, Leeds General Infirmary, Leeds, UK; ${ }^{2}$ Leeds Institute of Molecular Medicine, University of Leeds, Leeds, UK

Introduction Irritable bowel syndrome (IBS) and gastro-esophageal reflux disease (GERD) are common disorders in the general population, and there is thought to be a degree of overlap between the two. However, the strength of this association has not been examined systematically. We performed a systematic review and metaanalysis to estimate the prevalence of GERD in individuals with IBS. Methods MEDLINE, EMBASE, and EMBASE Classic were searched through October 2011 for cross-sectional surveys reporting the prevalence of IBS. A recursive search of the bibliographies of identified articles was also conducted. There were no language restrictions. Eligible studies were population-based, recruited $\geq 50$ adult ( $\geq 15$ years) subjects, and were required to define IBS via the Manning or Rome criteria, or according to a questionnaire. Eligibility assessment and data extraction were conducted independently in a double-blind fashion by two investigators, with any discrepancies resolved by consensus. Data were extracted and pooled, with a random effects model, to estimate prevalence of IBS in the population under study. The prevalence of GERD in individuals with and without IBS was compared using an OR, with a 95\% CI. Results The search yielded 20146 results, of which 390 studies appeared relevant and were retrieved for further assessment. There were 80 separate population-based studies that reported the prevalence of IBS according to the various criteria defined above. Of these, 13 studies, containing 49939 participants, also reported the proportion of people who met criteria for GERD within the same population. The pooled prevalence of IBS in these 13 studies was $11.6 \%$ (95\% CI $7.1 \%$ to $17.1 \%$ ). The odds of GERD in individuals with IBS, compared with those without, was 4.17 (95\% CI 2.85 to 6.09). Odds of GERD in individuals with IBS varied according to the criteria used to define IBS (see Abstract PWE-059 table 1).

\section{Abstract PWE-059 Table 1}

\begin{tabular}{lccl}
\hline $\begin{array}{l}\text { Definition of } \\
\text { IBS used }\end{array}$ & $\begin{array}{l}\text { Number of } \\
\text { studies }\end{array}$ & $\begin{array}{l}\text { Number of } \\
\text { subjects }\end{array}$ & OR (95\% CI) \\
\hline Manning & 3 & 5708 & $3.83(1.22$ to 11.97$)$ \\
Rome I & 5 & 6580 & $3.53(2.05$ to 6.10$)$ \\
Rome II & 3 & 18470 & $4.63(2.05$ to 10.44$)$ \\
Rome III & 1 & 18180 & $3.44(2.57$ to 4.59$)$ \\
Questionnaire & 1 & 1001 & $9.59(7.14$ to 12.87$)$ \\
Any definition & 13 & 49939 & $4.17(2.85$ to 6.09$)$ \\
\hline
\end{tabular}

Conclusion The prevalence of GERD in individuals with IBS was fourfold that in those without. The degree of overlap varied according to the criteria used to define IBS, but remained significant in all cases. The strength of this association suggests common pathogenetic mechanisms.

Competing interests None declared.

\section{PWE-060 EFFECT OF GENDER ON PREVALENCE AND SUBTYPE OF IRRITABLE BOWEL SYNDROME: A SYSTEMATIC REVIEW AND META-ANALYSIS}

doi:10.1136/gutjnl-2012-302514d.60

${ }^{1} \mathrm{R}$ M Lovell, ${ }^{* 1,2} \mathrm{~A}$ C Ford. ' ${ }^{1}$ Leeds Gastroenterology Institute, Leeds General Infirmary, Leeds, UK; ${ }^{2}$ Leeds Institute of Molecular Medicine, University of Leeds, Leeds, UK

Introduction It has always been assumed that irritable bowel syndrome (IBS) is more common in women. However, there has been no systematic review and meta-analysis that has synthesised data from all available studies to estimate prevalence of IBS according to gender. There has also been no study that has investigated whether the presumed higher prevalence in women varies according to the way in which IBS is defined, and whether it holds true for all IBS subtypes.

Methods MEDLINE, EMBASE, and EMBASE Classic were searched through October 2011 for cross-sectional surveys reporting the prevalence of IBS. A recursive search was also conducted. There were no language restrictions. Eligible studies were population-based, recruited $\geq 50$ adult ( $\geq 15$ years) subjects, and were required to define IBS via the Manning or Rome I, II or III criteria. Eligibility assessment and data extraction were conducted independently in a double-blind fashion by two investigators, with discrepancies resolved by consensus. Data were extracted and pooled, with a random effects model, to estimate prevalence of IBS according to gender. An OR, with a $95 \% \mathrm{CI}$, was used to compare the female to male prevalence of IBS, as well as the prevalence of constipationpredominant (IBS-C), diarrhoea-predominant (IBS-D), and mixedtype (IBS-M) among women and men meeting criteria for IBS.

Results The search yielded 20146 results, of which 390 studies appeared relevant and were retrieved for further assessment. There were 80 separate population-based studies that reported the prevalence of IBS according to the above criteria. Of these, 55 reported IBS prevalence according to gender, with a pooled prevalence in women of $14.0 \%(95 \%$ CI $11.0 \%$ to $16.0 \%)$ compared with $8.9 \%(95 \% \mathrm{CI}$ $7.3 \%$ to $10.5 \%$ ) in men (OR $1.67 ; 95 \%$ CI 1.53 to 1.82 ). Prevalence was consistently higher in women when all definitions of IBS were considered: the OR for women compared to men was $1.55(95 \% \mathrm{CI}$ 1.35 to 1.78 ) with the Manning criteria, 1.99 (95\% CI 1.76 to 2.25) with Rome I, 1.40 (95\% CI 1.24 to 1.59) with Rome II, and 1.81 (95\% CI 1.36 to 2.39 ) with Rome III. Nine studies, with 63827 participants, also reported the breakdown of IBS according to subtype. Prevalence of IBS-C was significantly higher in women with IBS compared with men (OR 2.38; $95 \%$ CI 1.45 to 3.92 ), IBS-D was less common in women with IBS compared with men (OR 0.45 ; $95 \%$ CI 0.32 to 0.65 ), while prevalence of IBS-M was not significantly different according to gender (OR 1.07; 95\% CI 0.84 to 1.38).

Conclusion Prevalence of IBS was modestly increased in women This observation remained stable according to the various diagnostic criteria used. However, among individuals with IBS, women were more likely to have IBS-C than men, and less likely to have IBS-D. These data suggest that gender may influence IBS subtype.

Competing interests None declared. 